\title{
Análise das relações entre o grau de complexidade das estruturas das cadeias de suprimento e o uso de indicadores de desempenho
}

\author{
Analysis of the relations between the degree of \\ complexity of supply chain structures and use \\ patterns of performance Indicators
}

\author{
Renata Porto Chaves ${ }^{1}$ \\ Antônio André Cunha Callado ${ }^{2}$
}

\section{Resumo}

A mensuração do desempenho tem sido amplamente investigada no âmbito de organizações individuais, mas pouca atenção tem sido dada à sua contextualização em cadeias de suprimento. Este artigo investiga as relações entre o grau de complexidade das estruturas das cadeias de suprimento e o uso de indicadores de desempenho. Para a realização desta pesquisa, foi analisada uma amostra composta por 36 organizações individuais participantes de cadeias de suprimento localizadas no estado de Pernambuco. Foram considerados 36 indicadores de desempenho, distribuídos entre cinco dimensões distintas (custos, custos logísticos, qualitativos, capacidade de resposta ao consumidor e desempenho do fornecedor). O instrumento utilizado para a operacionalização da coleta de dados foi um questionário. Para investigar a significância estatística das relações existentes entre as variáveis consideradas, foi utilizada a prova exata de Fisher. Os resultados obtidos apontam evidências que indicam a presença de algumas relações estatisticamente significativas entre os diversos indicadores de desempenho considerados, a saber: inventário e giro de estoque (custos logísticos), flexibilidade (qualitativos), tempo de processamento do pedido e quebra de estoques (capacidade de resposta ao

Mestre em Administração e Desenvolvimento Rural. Universidade Federal Rural de Pernambuco. Endereço: Rua 48, 165, apto. 103, Bairro do Espinheiro. Recife-PE. CEP: 50020-060. Fone: (081) 3320-6449. E-mail: portorena@hotmail.com.

2 Pós-Doutor em Controladoria pela University of Portsmouth, Doutor em Administração dela Universidade Federal de Pernambuco e pela Universidade Federal da Paraíba. Universidade Federal Rural de Pernambuco. Av. D. Manoel de Medeiros, s/n. Dois Irmãos. Recife-PE. CEP 52.171-030. Fone (081) 3320-6449. E-mail: andrecallado@yahoo.com.br. 
consumidor). Nenhum dos indicadores pertencentes às dimensões de custos e de desempenho do fornecedor se mostrou relacionado de maneira significativa com o grau de complexidade das relações das cadeias.

Palavras-chave: Controladoria. Mensuração de Desempenho. Desempenho de Cadeias de Suprimento.

\section{Abstract}

The performance measurement has been widely investigated in the context of individual organizations, but little attention has been given to its contextualization in supply chains. This article investigates the relationship between the complexity of supply chain structures and the use of supply chain performance indicators. For this research, a sample of 36 individual organizations that are part of supply chains from Pernambuco State was investigated and 36 performance indicators distributed among five distinct dimensions (costs, logistics costs, quality, responsiveness to consumer and supplier performance) were considered. The instrument used for data collection was a questionnaire. The Fisher's Exact Test was used to investigate the statistical significance of the relationship between the variables considered. The results show evidence that indicate the presence of some statistically significant relationships between several performance indicators considered, namely: inventory and inventory turnover (logistics costs), flexibility (qualitative); processing time of the application and breaks inventory (capacity response to the consumer). None of the indicators from cost and supplier performance dimensions were significantly related to the degree of complexity of the relationships of the chains.

Keywords: Controlling. Performance indicators. Supply Chain Performance.

\section{Introdução}

A mudança do paradigma competitivo, baseada na competição entre cadeias de suprimentos, e não mais entre empresas isoladas, fez com que a preocupação com a avaliação do desempenho ultrapassasse as fronteiras físicas das empresas e englobasse a avaliação da cadeia como um todo, com o objetivo principal de melhorar o posicionamento competitivo da referida cadeia. $O$ desenvolvimento e a implementação de sistemas de mensuração de desempenho de cadeias requeridos para dar suporte a uma efetiva gestão da cadeia deveriam ir além das fronteiras das empresas individuais e cobrir a cadeia como um todo (LUCHT, 2005). 
De acordo com Lovejoy e Tsay (1999), muitas cadeias de suprimentos operam sob uma perspectiva de terceirização (outsourcing) de diversos aspectos relativos ao processo produtivo. Esse fenômeno faz com que haja uma descentralização do poder decisório e a difusão de que ações e decisões individuais causam impactos diretos na performance global.

No caso das cadeias de suprimentos, composta por agentes independentes, alcançar melhorias globais não é algo fácil, em função, principalmente, da incongruência entre os problemas de desempenho individuais e os problemas da otimização global. No entanto, no contexto da cadeia, segundo Gasparetto (2003), a busca de melhorias individuais envolve ações e decisões que dependem de mudanças em processos que estão fora das fronteiras das empresas e, nessa perspectiva, outras empresas têm importante participação no sucesso de tais esforços.

Tradicionalmente, a atenção dada para a mensuração de desempenho de cadeias de suprimento tem sido canalizada para processos específicos presentes ao longo de toda a estrutura da cadeia. Recentemente, mais ênfase tem sido dada para a mensuração do desempenho global das cadeias (RAFELE, 2004). A literatura não apresenta nenhum consenso sobre um conjunto de indicadores relevantes para avaliar o desempenho de uma cadeia de suprimento. Para Fan, Najmi, Rigas (2005), a identificação das características desejáveis para os sistemas de mensuração de desempenho de cadeias de suprimento tem se tornado uma meta tanto para acadêmicos quanto para profissionais de mercado.

No contexto das cadeias de suprimento, desempenho não tem sido considerado um fator referente a uma única empresa, e sim o desempenho de todas as empresas participantes como responsáveis pelo desempenho global da cadeia (CHEN; PAULRAJ, 2003), bem como influenciam umas as outras e afetam o desempenho das demais empresas individuais (BIGLIARDI; BOTTANI, 2010).

Dada a complexidade inerente à gestão da cadeia de suprimento, a capacidade de mensurar o desempenho de seus diversos aspectos 
operacionais assume uma importância crítica, principalmente considerando que o elenco de indicadores para mensurar o desempenho a ser utilizado deve ser escolhido considerando a natureza das organizações (ELROD; MURRAY; BANDE, 2013), e que o tamanho e a complexidade das cadeias podem afetar seu desempenho (MENA; HUMPHRIES; CHOI, 2013). Para Alcântara e Pigatto (2006), a complexidade das estruturas das cadeias de suprimentos é um fator que influencia diretamente o seu desempenho. $\mathrm{O}$ objetivo deste artigo é investigar as relações entre o grau de complexidade das estruturas das cadeias de suprimento e o uso de indicadores de desempenho.

\section{Gestão de cadeias de suprimento}

A expressão "cadeia de suprimento" foi apresentada pela primeira vez na literatura por Oliver e Webber (1982). Desde então, essa expressão tem sido usada para representar atividades de planejamento e de controle dos fluxos de materiais e de informação, bem como as atividades de logística interna e externa inerentes a cadeias de suprimento (COOPER; LAMBERT; PAGH, 1997). A gestão de cadeia de suprimento surgiu originalmente, tanto na literatura acadêmica quanto no âmbito da prática dos negócios, com a finalidade de reunir esforços para reduzir grandes quantidades de estoques que se acumulavam pelo canal e que outros objetivos foram sendo buscados pelo gerenciamento de uma cadeia de suprimento, tais como:

- Redução de investimento em estoques no canal;

- Aumentar o nível de serviço oferecido aos clientes finais;

- Ajudar a construir vantagens competitivas para os membros da cadeia.

(COOPER; ELLRAM, 1993)

A redução de investimento em estoque no canal envolve um esforço significativo das empresas individuais e dos diversos processos operacionais desempenhados no âmbito das cadeias de suprimentos. Essa redução representa uma economia significativa para a cadeia, 
bem como para as empresas participantes, e pode ser feita por meio de uma gestão eficaz de inventário ao longo de toda estrutura da cadeia, através de uma coordenação sincronizada do suprimento e da gestão de estoques.

O aumento do nível de serviços oferecidos aos consumidores finais está relacionado à redução de investimentos em estoques, uma vez que a elevação do nível dos serviços prestados dependerá da eliminação de estoques desnecessários, bem como da manutenção de estoques de segurança suficientes para atender os níveis de serviços esperados. Para isso, o compartilhamento de informações consistentes referentes à demanda prevista é fundamental para a realização de projeções confiáveis e apropriadas para o atendimento das reais necessidades dos clientes.

Com relação ao compartilhamento de informações que devem ocorrer ao longo da estrutura da cadeia, três observações relevantes se fazem necessárias. Em primeiro lugar, a literatura indica que, para uma efetiva gestão da cadeia de suprimentos, faz-se necessário o compartilhamento constante de informações. Essa medida visa aumentar o nível de conhecimento sobre a demanda da cadeia, além de melhorar o grau de precisão da projeção da demanda e reduzir a incerteza (CONCEIÇÃO; QUINTÃO, 2004). É importante salientar que essas informações não só são geradas a partir de dados acerca dos clientes coletados, elas dão suporte ao planejamento das vendas, bem como correspondem a um fluxo bidirecional de troca de informações entre clientes e fornecedores ao longo da cadeia. Em segundo lugar, o caminho tradicionalmente percorrido pela informação relacionada à demanda por um produto ou serviço no interior da cadeia assume uma importância gerencial relevante. O que se observa, muitas vezes, é que as informações fluem de forma desconexa, e isso ocorre, principalmente, devido ao fato de que cada elo mantém suas informações relacionadas a níveis de estoque, projeção de vendas e planejamento de distribuição no âmbito organizacional, e não as compartilham com as outras empresas pertencentes à cadeia (BATALHA; SCRAMIM, 1999). Em terceiro lugar, o aumento do nível dos serviços está relacionado aos dois objetivos 
principais do compartilhamento de informações entre os elos pertencentes à cadeia. Segundo Batalha e Scramin (1999), essas informações devem ser compartilhadas entre os membros da cadeia, a fim de que seja obtido feedback de como a performance de uma empresa participante está sendo percebida pelas demais, bem como pelos consumidores finais. O compartilhamento de informações é importante tanto para a redução de investimento em estoque quanto para o melhoramento contínuo do nível de serviço oferecido ao cliente, na perspectiva definida por Cooper e Ellram (1993).

A contribuição para a construção de vantagens competitivas para os membros da cadeia é operacionalizada a partir da redução de estoque, que auxilia na minimização de custos totais de produção, o que torna os preços dos produtos mais competitivos. Outro aspecto associado à construção de vantagens competitivas é o aumento do nível de serviço oferecido aos clientes, decorrente do suporte gerencial dado através de um fluxo adequado de informações entre as empresas participantes.

Outro aspecto relevante para o entendimento teórico acerca da gestão da cadeia de suprimento se refere às relações compradorfornecedor. Para Chen e Paulraj (2003), essas relações abrangem cinco características, a saber:

- Redução da base de fornecedores

- Relações de longo prazo

- Comunicação

- Equipes multifuncionais

- Envolvimento dos fornecedores

As relações entre compradores e fornecedores passaram a receber maior atenção por parte dos gestores à medida que a noção de que relações comerciais de sucesso teriam maior probabilidade de ocorrer a partir da existência de cooperação mútua foi difundido. 0 gerenciamento tradicional baseado no poder de barganha e nas relações de enfrentamento abriu espaço para uma nova concepção de gestão orientada pela cooperação e pela integração (BROWERSOX; CLOSS, 2001). 
De acordo com Ballou, Gilbert e Mukherjee (2000), a gestão da cadeia de suprimento abrange três dimensões distintas:

- Coordenação intrafuncional

- Coordenação de atividades interfuncionais

- Coordenação de atividades interorganizacionais da cadeia de suprimento

A primeira se refere à administração de atividades e processos dentro das funções logísticas das empresas. A segunda se refere à administração de atividades entre distintas áreas funcionais das empresas. A terceira se refere à administração de atividades entre distintas empresas que fazem parte da cadeia.

Ao longo das duas últimas décadas, a gestão da cadeia de suprimento tem enfatizado a interdependência de empresas compradoras e fornecedoras que atuam de maneira colaborativa, com a finalidade de aprimorar o desempenho da cadeia como um todo (SHIN; COLLIER; WILSON, 2000).

A gestão de componentes gerenciais da cadeia, tais como estrutura de trabalho (conjunto de tarefas e atividades executadas pelas empresas), estrutura do fluxo de produtos (abastecimento, produção e distribuição ao longo da cadeia), planejamento e controle das operações, são considerados por Pires (2004) como elementos importantes para o processo de integração da cadeia.

As interdependências entre as empresas pertencentes a uma cadeia de suprimento fizeram com que acadêmicos e profissionais de mercado buscassem a identificação de meios de coordenar as operações de negócios, bem como as relações entre as empresas que a compõem com o objetivo de integrar os processos chaves associados aos diferentes elos em busca de vantagens competitivas sustentáveis em relação a outras cadeias concorrentes. Nesse contexto, a gestão da cadeia de suprimento assume o papel de modelo de gestão que busca superar os conflitos tradicionais existentes entre as empresas e promovem integrações efetivas para alcançar uma performance conjunta superior. 
Essa compreensão é cada vez mais importante dentro de um cenário em que as empresas mantêm vínculos diretos e a agregação de valor ao produto ou ao serviço é operacionalizada a partir de um conjunto de atividades e processos estratégicos importantes desempenhados por diferentes empresas. Desse modo, há uma expectativa de que o estabelecimento de relações de cooperação possa aumentar significativamente a competitividade de todas as empresas individuais envolvidas e que uma gestão de cadeia voltada para o alcance de objetivos estratégicos comuns represente o fortalecimento de elementos competitivos importantes.

Segundo Cooper e Ellram (1993), a gestão da cadeia de suprimento aborda a integração vertical de cooperação nos distintos níveis de uma cadeia, através do compartilhamento de informações, tecnologia, infraestrutura e habilidades, em busca de padrões de qualidade e especificações requeridas pelos consumidores finais. Nesse tipo de integração, uma empresa exerce um controle gerencial sobre a outra e a decisão referente à operacionalização dessa integração vertical decorre de fatores específicos, tais como economia de escala e disponibilização de serviços. As operações são realizadas através do controle exercido por uma empresa focal responsável pelo processo decisório referente às principais questões referentes à cadeia, bem como pala coordenação e gerenciamento da cadeia como um todo.

Outro aspecto relevante é a visualização da estrutura de relações existentes na cadeia, consistindo na identificação de cada uma das empresas individuais participantes, bem como na análise de suas dimensões estruturais. Segundo Pires (2004), as empresas participantes são as organizações com as quais a empresa focal da cadeia interage de maneira direta e indireta, a montante e a jusantes, desde os fornecedores de matérias-primas até os consumidores finais.

As empresas individuais que compõem uma cadeia de suprimento podem ser classificadas como participantes primários e participantes de suporte. Os participantes primários são as empresas que atuam de maneira direta no processo de agregação de valor ao produto 
considerando as exigências e necessidades de determinados clientes ou mercados (PIRES, 2004). Por outro lado, os participantes de suporte são responsáveis pelo fornecimento de conhecimentos, facilidades ou ativos para os participantes primários da cadeia. Vale salientar que os participantes a serem considerados quando do mapeamento da estrutura de uma cadeia de suprimento e que requerem atenção no seu gerenciamento devem ser aqueles considerados críticos para o sucesso dela e que a identificação dos participantes primários indicam aos demais focalizarem esforços nos relacionamentos e interações realmente, agregam valor ao produto ou serviço (MORAIS, 2008).

Uma mesma empresa pode desempenhar mais de um papel dentro da estrutura de uma cadeia de suprimentos, atuando como participante de suporte em um processo operacional específico e como participante primária em outro. Pires (2004) nos fornece o seguinte exemplo: uma empresa montadora pode trabalhar de maneira conjunta com um fornecedor específico no processo de desenvolvimento de um equipamento para a produção de um novo produto. Nesse caso, o fornecedor atua como participante primário, no entanto, a partir do momento que o equipamento é finalizado e ingressa no processo produtivo da montadora, o fornecedor passa a ser um participante de suporte.

O grau de complexidade de uma cadeia de suprimento pode estar associado tanto ao número de níveis (echelons) ao longo do fluxo de materiais e informações quanto ao número de empresas em cada um dos níveis (Beamon, 1998). Para Callado e Callado (2010), a disposição sequencial dos distintos níveis que integram o fluxo de atividades da cadeia representa sua estrutura vertical, enquanto o conjunto de empresas que atuam em cada um dos níveis integra estruturas horizontais.

Além disso, a implantação de qualquer modelo de integração da cadeia de suprimento poderá sofrer barreiras significativas em função, principalmente, dos diferentes interesses envolvidos individuais de cada uma das empresas envolvidas. No entanto, em um cenário em que o paradigma competitivo assume que a competição ocorra entre cadeias 
e não mais entre empresas individuais isoladamente, emerge cada vez mais a importância de um gerenciamento efetivo dos processos e atividades da cadeia, bem como de ferramentas para monitorá-lo tais como os sistemas de mensuração de desempenho de cadeias de suprimento.

\section{Mensuração do desempenho de cadeias de suprimento}

Para uma efetiva gestão de cadeia de suprimentos, faz-se necessária a implantação de ferramentas gerenciais que possam monitorar e controlar seus participantes, bem como os processos e atividades desenvolvidas ao longo de sua estrutura. A avaliação de desempenho é uma temática que já algum tempo vem adquirindo importância no âmbito empresarial e acadêmico, e a partir da disseminação das teorias referentes à cadeia suprimento houve uma preocupação em estudar sistemas de mensuração que englobe o conjunto de empresas pertencentes a cadeias de suprimento.

A mensuração de desempenho pode ser definida, então, como o processo de quantificar a ação, em que a mensuração é a maneira de quantificar e ação está relacionada ao desempenho, podendo envolver duas dimensões: eficiência e eficácia. A eficiência está relacionada aos parâmetros econômicos de utilização dos recursos necessários para alcançar a satisfação dos clientes em relação aos produtos vendidos ou aos serviços prestados. Já a eficácia refere-se ao alcance dessa satisfação. Nesse sentido, mensuração de desempenho pode ser também definida como o processo de quantificar a eficiência e eficácia de uma ação (NEELY; GREGORY e PLATTS, 1995).

Segundo Bond (2002), mensuração de desempenho pode ser definida, de maneira genérica, como a atividade de se determinar as medidas de desempenho no sentido de adequar, ajustar e controlar alguma atividade. Vale destacar que os objetivos da análise de desempenho podem variar de acordo com as necessidades específicas de cada empresa, entretanto, pode-se afirmar que o objetivo central de 
uma medição de desempenho é apontar se as empresas estão seguindo o caminho certo para alcançar as metas estrategicamente determinadas.

De acordo com Nelly, Gregory e Platts (1995), mensuração de desempenho, sistemas de mensuração de desempenho e indicadores de desempenho possuem significados próprios. O primeiro se refere a processos de quantificação acerca de determinada ação, em termos de eficiência e eficácia. O segundo se refere a um conjunto amplo de medidas que servem para avaliar processos e atividades desenvolvidas. O terceiro se refere a medidas quantificáveis utilizadas para mensurar a eficácia e eficiência de uma ação.

No caso das cadeias de suprimento, a mensuração de desempenho é algo mais complexo, já que elas representam um conjunto de empresas com um aglomerado de processos e atividades interligados. A literatura destaca que é difícil estabelecer um conjunto de indicadores adequados que representem todos os processos e atividades-chave de uma cadeia, já que o desempenho da cadeia depende, ao mesmo tempo, da performance individual dos elos e das ações conjuntas executadas por eles.

Rafele (2004) afirma que, anteriormente, a mensuração de desempenho era voltada para processos específicos da cadeia de suprimento, no entanto, a partir do final da década de 1990, o foco tem sido dado à avaliação da cadeia de maneira global. Nessa perspectiva, o desenvolvimento de um sistema de mensuração para a cadeia de suprimento é fundamental para que a gestão ultrapasse o limite das fronteiras das empresas e alcance o sistema como um todo (LUCHT, 2005).

O desenvolvimento de sistemas de mensuração para cadeias leva em consideração o processo de seleção das métricas adequadas. Dentro da literatura, não há consenso acerca dos critérios a serem considerados no processo de seleção de indicadores para a mensuração do desempenho para cadeias de suprimento. O que se considera como consenso é que não existe um conjunto pré-estabelecido de medidas que seja aplicável a qualquer cadeia de suprimento, já que cada cadeia 
possui características próprias que requerem indicadores diferentes (BEAMON, 1999).

De acordo com Beamon (1998), um importante componente no projeto de uma cadeia de suprimento é a implantação de um sistema de mensuração de desempenho que deve considerar indicadores de desempenho consistentes para avaliar a performance considerando medidas qualitativa e quantitativa.

As medidas quantitativas relacionadas, principalmente, ao desempenho financeiro são aquelas que podem ser descritas diretamente, de maneira numérica. Gunasekaran, Patel e McGauguey (2004) afirmam que uma cadeia de suprimentos deve ser avaliada através de medidas financeiras relacionadas aos custos logísticos e de produção, uma vez que elas possuam forte impacto nas estratégias e práticas ligadas ao fluxo de materiais e informações. Já as medidas qualitativas são métricas para as quais não existem números diretos para medição, destacando a satisfação do cliente e flexibilidade. O primeiro representa o grau de satisfação do cliente em relação aos produtos vendidos ou aos serviços prestados, enquanto o segundo reflete a capacidade que uma cadeia possui para responder aos consumidores no atendimento de suas necessidades e especificações.

Em outra vertente, Gunasekaran, Patel e McGaughey (2004) apresentam um conjunto de indicadores relacionados a quatro dimensões funcionais das cadeias de suprimento: planejamento, fornecimento, produção e entrega. O planejamento está ligado a um elenco de medidas que reflitam as formas como as especificações e necessidades dos clientes são convertidas em ações e informações no interior da cadeia (um exemplo de indicador pertencente a essa categoria é o tempo de pedido). Sobre fornecimento, os autores ressaltam que durante muito tempo a seleção de fornecedores foi baseada na concorrência de preços, no entanto, no contexto da cadeia de suprimento, considerando qualidade e confiabilidade. Com relação à produção, os autores apontam qualidade, custos operacionais, velocidade de entrega, flexibilidade e confiabilidade como categorias de indicadores que devem ser consideradas. Para as 
atividades de entrega, eles destacam que a satisfação dos consumidores e as atividades de pós-venda devem ser monitoradas.

Segundo Holfman (2004), a preocupação na avaliação de desempenho de uma cadeia de suprimento se concentra na definição de indicadores que realmente importam para avaliar a cadeia. Uma estrutura de indicadores de uma cadeia, portanto, deve levar em consideração suas especificidades, tais como: número de empresas individuais envolvidas, processos de negócios desenvolvidos e arranjos de relações estabelecidos entre as empresas participantes.

\section{Procedimentos metodológicos}

A pesquisa foi realizada junto às empresas localizadas na Região Metropolitana de Recife, no estado de Pernambuco. No caso deste trabalho, foi definida uma amostra composta por 90 empresas que trabalham com produtos agrícolas localizadas na Região Metropolitana do Recife, selecionadas pelo critério de acessibilidade. Todas as 90 empresas selecionadas foram contatadas com o intuito de verificar sua disponibilidade em participar da pesquisa e 38 aceitaram participar da pesquisa. Destas, apenas 36 se disponibilizaram em fornecer os dados necessários para a operacionalização da pesquisa. Essa amostra é formada por empresas produtoras e distribuidoras do varejo e atacado (Tabela 1). 
Tabela 1: Perfil das empresas que compõem a amostra

\begin{tabular}{lcc}
\hline Empresas & Segmento de atuação & Amostra \\
\hline Produtores & Alimentos & 12 \\
Produtores & Outros & 1 \\
Varejo & Alimentos & 15 \\
Atacado & Alimentos & 5 \\
Atacado & Outros & 3 \\
\hline Total & & 36 \\
\hline
\end{tabular}

A investigação sobre os padrões de uso de indicadores de desempenho de cadeia foi operacionalizada a partir de 36 indicadores distribuídos entre cinco grupos distintos, a saber:

- Custos (BEAMON, 1998): minimização de custos, maximização de vendas, maximização de lucros, inventário sobre o investimento, retorno sobre o investimento, custo total de produção, tempo do produto em estoque.

- Custos logísticos (BROWERSOX; CLOSS, 2001): custos de armazenagem, custos de transporte, inventário, custos do pedido e giro de estoque.

- Qualitativos (RAFELE, 2004; GUNASEKARAN; PATEL; McGAUCHEY, 2004): satisfação do consumidor, flexibilidade, informação e integração de materiais, gestão do risco, responsividade, crescimento de market share e gestão da inovação.

- Capacidade de resposta ao consumidor (BEAMON, 1998): tempo de processamento do pedido, tempo de entrega, flexibilidade, devoluções, produtividade do pessoal, satisfação do consumidor e quebra de estoque.

- Desempenho do fornecedor (CONCEIÇÃO; QUINTÃO, 2004): tempo de recebimento, recebimento de acordo com as especificações exigidas, devoluções parcial ou integral de produtos ao fornecedor, entregas realizadas no prazo e atendimento do produto realizado.

O grau de complexidade das cadeias de suprimento foi abordado a partir das características das estruturas de relações. Estruturas simples 
são caracterizadas pelo fluxo sequencial linear entre os participantes da cadeia, enquanto estruturas complexas são caracterizadas pela presença de fluxos cruzados.

Para a coleta de dados nesta pesquisa, foi utilizado um questionário composto por perguntas fechadas. De acordo com Gil (2008), um questionário serve para quantificar um determinado fenômeno com finalidades específicas, tais como classificação e análise de resultados. Ainda em relação às vantagens do questionário, Goldenberg (2002) afirma que esse instrumento é menos dispendioso, exige menor habilidade de aplicação, pode ser enviado ou entregue em mão, pode ser aplicado a um grande número de pessoas ao mesmo tempo, bem como proporciona uma padronização de respostas assegurando maior uniformidade.

Das 36 empresas que constituem a amostra, a quase totalidade delas (34 empresas) entregou o questionário impresso e preenchido pessoalmente, enquanto outras 2 empresas enviaram o questionário respondido via e-mail. Todos os procedimentos relativos à coleta dos dados foram realizados no período de 01/08/2011 a 31/10/2011.

Em função das características da amostra utilizada nesta pesquisa, não foi possível utilizar o teste paramétrico qui-quadrado, optando-se pela prova exata de Fischer. Levin (1987) destaca que a prova exata de Fischer permite resolver o problema de significância, quando a amostra não possui distribuição normal das variáveis investigadas, e dessa forma, não fornece ao pesquisador os parâmetros paramétricos clássicos, tais como: medidas centrais e de variância. O nível de significância considerado como aceitável foi de $95 \%(p=0,05)$. Todos os procedimentos foram realizados através do auxílio do aplicativo estatístico Statistica for Windows.

\section{Resultados}

A partir do objetivo proposto e dos procedimentos metodológicos adotados, foi operacionalizada a análise estatística dos dados, em 
busca dos resultados referentes à significância estatística das relações entre o uso dos indicadores de desempenho considerados e o grau de complexidade das estruturas das cadeias. O primeiro grupo de relações envolve os indicadores de desempenho pertencentes ao grupo de custos. Os resultados referentes à significância das relações existentes entre o uso dos indicadores de custos e o grau de complexidade das estruturas das cadeias estão dispostos na tabela 2.

Tabela 2 - Resultados da prova exata de Fischer referentes às relações entre o grau de complexidade das relações na cadeia e o uso dos indicadores de custos

\begin{tabular}{lc}
\hline Indicadores de custos & $\mathbf{P}$ \\
\hline Minimização de custos & 0,45 \\
Maximização de vendas & 0,17 \\
Maximização de lucros & 0,15 \\
Inventário de investimentos & 0,15 \\
Retorno sobre investimento & 0,21 \\
Custo total da produção & 0,22 \\
Tempo do produto em estoque & 0,57 \\
\hline
\end{tabular}

Através dos resultados obtidos, pode-se observar que nenhum dos indicadores de custos considerados apresentou padrões de uso relacionados ao grau de complexidade das cadeias de suprimento maneira significativa. O segundo grupo de relações envolve os indicadores de desempenho pertencentes ao grupo de custos logísticos e o grau de complexidade das cadeias. Os resultados estão apresentados na Tabela 3. 
Tabela 3 - Resultados da prova exata de Fischer referentes às relações entre o grau de complexidade das relações na cadeia e o uso dos indicadores de custos logísticos

\begin{tabular}{ll}
\hline Indicadores de custos logísticos & $\mathbf{P}$ \\
\hline Custo total de produção & 0,22 \\
Custo de armazenagem & 0,11 \\
Custo de transporte & 0,08 \\
Inventário & 0,00 \\
Retorno sobre o investimento & 0,65 \\
Custo de pedido & 0,43 \\
Giro de estoque & 0,04 \\
\hline
\end{tabular}

Pode-se observar através dos resultados obtidos que o uso de dois dos indicadores de desempenho testados (inventário e giro dos estoques) se mostrou relacionado ao grau de complexidade das cadeias de maneira estatisticamente significativa. Para proporcionar uma melhor visualização das características dessas relações, foram elaboradas tabelas de distribuição condicional de frequência. Na Tabela 4, está disposta a estrutura de relações entre o uso do indicador de desempenho referente ao uso de inventários e o grau de complexidade das cadeias.

Tabela 4 - Distribuição condicional de frequência do uso do indicador de desempenho referente a inventário em função do grau de complexidade das cadeias de suprimento

\begin{tabular}{lcc}
\hline & $\begin{array}{c}\text { Cadeias de estruturas } \\
\text { simples }\end{array}$ & $\begin{array}{c}\text { Cadeias de estruturas } \\
\text { complexas }\end{array}$ \\
\hline Não usa & 14 & 2 \\
Usa & 8 & 12 \\
\hline Total & 22 & 14 \\
\hline
\end{tabular}

Pode-se observar que a quase totalidade das empresas que declararam pertencer a cadeias que possuem relações complexas informaram que usam este indicador, enquanto a maior parte das empresas que declarou pertencer a cadeias que possuem estruturas de 
relações simples informou que não o usa. Do mesmo modo, a estrutura das relações entre o uso do indicador de desempenho referente ao giro de estoque e o grau de complexidade das cadeias está apresentada na Tabela 5.

Tabela 5 - Distribuição condicional de frequência do uso do indicador de desempenho referente ao giro de estoque em função do grau de complexidade das cadeias de suprimento

\begin{tabular}{lcc}
\hline & $\begin{array}{c}\text { Cadeias de estruturas } \\
\text { simples }\end{array}$ & $\begin{array}{c}\text { Cadeias de estruturas } \\
\text { complexas }\end{array}$ \\
\hline Não usa & 14 & 4 \\
Usa & 8 & 10 \\
\hline Total & 22 & 14 \\
\hline
\end{tabular}

Os resultados obtidos se assemelham aos resultados apresentados na Tabela 4. Verificou-se que a maioria das empresas que declarou pertencer a cadeias que possuem estruturas complexas informou utilizar este indicador, enquanto quase dois terços das empresas que declararam pertencer a cadeias que possuem estruturas simples informaram que não o usam. O terceiro grupo de indicadores de desempenho testado se refere aos indicadores qualitativos. Os resultados estão dispostos na Tabela 6.

Tabela 6 - Resultados da prova exata de Fischer referentes às relações entre o grau de complexidade das relações na cadeia e o uso dos indicadores qualitativos

\begin{tabular}{lc}
\hline Indicadores qualitativos & $\mathbf{p}$ \\
\hline Satisfação dos consumidores & 0,61 \\
Flexibilidade & 0,02 \\
Informação e integração de materiais & 0,43 \\
Gestão do risco & 0,63 \\
Desempenho do fornecedor & 0,62 \\
Responsividade & 0,43 \\
Crescimento de market share & 0,36 \\
Gestão da inovação & 0,49 \\
\hline
\end{tabular}


Os resultados obtidos demonstram que apenas o indicador de desempenho referente à flexibilidade se mostrou relacionado ao grau de complexidade das cadeias de suprimento maneira estatisticamente significativa. Na Tabela 7, está disposta a estrutura de relações entre o uso do indicador de desempenho referente à flexibilidade e o grau de complexidade das cadeias.

Tabela 7 - Distribuição condicional de frequência do uso do indicador de desempenho referente à flexibilidade em função do grau de complexidade das cadeias de suprimento

\begin{tabular}{lcc}
\hline & $\begin{array}{c}\text { Cadeias de estruturas } \\
\text { Simples }\end{array}$ & $\begin{array}{c}\text { Cadeias de estruturas } \\
\text { complexas }\end{array}$ \\
\hline Não usa & 9 & 11 \\
Usa & 13 & 3 \\
\hline Total & 22 & 14 \\
\hline
\end{tabular}

Pode-se observar a presença de resultados contrários aos encontrados para inventário e giro dos estoques. Enquanto mais da metade das empresas que declararam participar de cadeias que possuem estruturas simples informou que usa indicador de desempenho referente à flexibilidade, a quase totalidade das empresas que declararam atuar em cadeias que possuem estruturas complexas informou que não usa este indicador de desempenho.

O quarto grupo de indicadores testados consiste na capacidade de resposta aos consumidores. Os resultados referentes às relações entre os indicadores desse grupo e o grau de complexidade das cadeias estão apresentados na Tabela 8. 
Tabela 8 - Resultados da prova exata de Fischer referentes às relações entre o grau de complexidade das relações na cadeia e o uso dos indicadores de capacidade de resposta ao consumidor

\begin{tabular}{lc}
\hline Indicadores de capacidade resposta ao consumidor & $\mathbf{P}$ \\
\hline Tempo de processamento do pedido & 0,02 \\
Tempo de entrega & 0,65 \\
Flexibilidade & 0,34 \\
Devoluções & 0,11 \\
Produtividade do pessoal & 0,56 \\
Satisfação do consumidor & 0,18 \\
Quebra de estoque & 0,02 \\
\hline
\end{tabular}

Os resultados obtidos apontam para a presença de relações estatisticamente significativas para dois dos indicadores de desempenho considerados nesse grupo (tempo de processamento de pedidos e quebra de estoque). Na Tabela 9, está disposta a estrutura de relações entre o uso do indicador referente ao tempo de processamento do pedido e o grau de complexidade das cadeias.

Tabela 9 - Distribuição condicional de frequência do uso do indicador de desempenho referente ao tempo de processamento do pedido em função do grau de complexidade das cadeias de suprimento

\begin{tabular}{lcc}
\hline & $\begin{array}{c}\text { Cadeias de estruturas } \\
\text { simples }\end{array}$ & $\begin{array}{c}\text { Cadeias de estruturas } \\
\text { Complexas }\end{array}$ \\
\hline Não usa & 9 & 1 \\
Usa & 13 & 13 \\
\hline Total & 22 & 14 \\
\hline
\end{tabular}

Observou-se que a frequência maior referente ao uso do indicador de desempenho referente ao tempo de processamento do pedido foi encontrada tanto entre empresas que participam em cadeias que possuem estruturas simples quanto entre empresas que atuam em cadeias com estruturas complexas. Entre as empresas que declararam pertencer a cadeias que possuem estruturas simples, foi encontrada 
uma ligeira prevalência acerco do uso deste indicador enquanto quase todas as empresas que declararam pertencer a cadeias que possuem estruturas complexas informaram que usam. Adotando o mesmo procedimento, a estrutura das relações entre o uso do indicador de desempenho referente à quebra de estoque e o grau de complexidade das cadeias está apresentada na Tabela 10.

Tabela 10 - Distribuição condicional de frequência do uso do indicador de desempenho referente à quebra do estoque em função do grau de complexidade das cadeias de suprimento

\begin{tabular}{lcc}
\hline & $\begin{array}{c}\text { Cadeias de estruturas } \\
\text { simples }\end{array}$ & $\begin{array}{c}\text { Cadeias de estruturas } \\
\text { complexas }\end{array}$ \\
\hline Não usa & 19 & 7 \\
Usa & 3 & 7 \\
\hline Total & 22 & 14 \\
\hline
\end{tabular}

Pode-se observar que a quase totalidade das empresas que declararam pertencer às cadeias de estrutura simples informou não usar esse indicador, enquanto entre as empresas que declararam que atuam em cadeias que possuem estruturas complexas não há prevalência.

O quinto grupo de relações envolve os indicadores de desempenho pertencentes ao grupo dos fornecedores e o grau de complexidade das cadeias. Os resultados estão apresentados na Tabela 11.

Tabela 11 - Resultados da prova exata de Fischer referentes às relações entre o grau de complexidade das relações na cadeia e o uso dos indicadores de desempenho do fornecedor

\begin{tabular}{lc}
\hline Indicadores baseados no desempenho do fornecedor & $\mathbf{p}$ \\
\hline Tempo de recebimento & 0,61 \\
Recebimento dos produtos de acordo com as especificações & 0,50 \\
exigidas & 0,27 \\
Devoluções parciais ou integral de produtos ao fornecedor & 0,21 \\
Entregas realizados no prazo & 0,11 \\
Atendimento do produto realizado & \\
\hline
\end{tabular}


Através dos resultados obtidos, observou-se que o uso de nenhum dos indicadores de custos considerados se mostrou relacionado às relações na cadeia de maneira significativa.

\section{Conclusão}

Este artigo buscou investigar as relações entre o grau de complexidade das estruturas das cadeias de suprimento e o uso de indicadores de desempenho. Para atingir o objetivo proposto, foi utilizada a prova exata de Fisher. Os resultados obtidos demonstraram a presença de algumas relações estatisticamente significativas.

Entre os cinco grupos de indicadores de desempenho de cadeia testados, custos e desempenho dos fornecedores não tiveram nenhum indicador relacionado de maneira significativa à complexidade da estrutura das cadeias, indicando que a relevância desses dois aspectos no contexto do gerenciamento das cadeias de suprimento independe do grau de complexidade das estruturas das cadeias. Para os outros três grupos testados, foram encontradas relações estatisticamente significativas, indicando prevalência do uso entre empresas participantes de cadeias que possuem estruturas simples para uns e prevalência de uso para outros e empresas que atuam em cadeias que possuem estruturas complexas.

Embora os resultados obtidos sejam estatisticamente significativos, eles não são suficientes para responder todas as questões relativas ao uso de indicadores de desempenho de cadeias de suprimento. Faz-se necessário o aprofundamento deste eixo investigativo dentro do novo paradigma de competitividade (baseada na competição entre cadeias de suprimentos e não mais entre empresas individuais). Nessa perspectiva, a avaliação do desempenho de cadeias poderá contribuir de maneira marcante para essa nova fronteira investigativa, principalmente lançando indícios sobre o conjunto de grupos indicadores de desempenho a ser considerado, da mesma maneira que aconteceu com o balanced scorecard para as empresas individuais. 
Outro eixo investigativo promissor se refere à complexidade das estruturas das cadeias de suprimentos. A diversidade de arranjos (decorrentes da grande quantidade de empresas e processos envolvidos, bem como da intensidade de trocas) é um elemento crucial a ser considerado nesse novo paradigma. Estudos mais aprofundados poderão gerar informações mais detalhadas sobre a natureza das relações dinâmicas encontradas nesta pesquisa.

Uma gestão de cadeia de suprimentos que considere indicadores de desempenho cadeia poderá dispor de instrumentos gerenciais importantes para a manutenção um posicionamento de mercado consistente a partir da busca contínua do aprimoramento de seu desempenho global.

\section{Referências}

BALLOU, R.H.; GILBERT, S.M.; MUKHERJEE, A. New managerial challenges from supply chain opportunities. Industrial Marketing Management, Cambridge, v. 29, n. 1, p. 7-18, Jan.uary, 2000.

BATALHA, M. O; SCRAMIM, F. C. L. Supply chain managament em cadeias agroindustriais: discussões a cerca das aplicações no setor lácteo brasileiro. In: WORKSHOP BRASILEIRO DE GESTÃO DE SISTEMA AGROALIMENTARES, 2., 1999, São Paulo. Anais... São Paulo, 1999. CD-ROM.

BEAMON, B. M. Measuring supply chain performance. International Journal of Operations and Production Management, Cambridge, v. 19, n. 3, p. 275-292, March, 1999.

Supply chain design and analysis: Models and methods. International Journal of Production Economics, Seatle, v. 55, n. 3, p. 281-294, August, 1998.

BIGLIARDI, B.; BOTTANI, E. Performance measurement in the food supply chain: a balanced scorecard approach. Facilities, Cambridge, v. 28, n. 5/6, p. 249-260, May-June, 2010. 
BOND, E. Medição de desempenho para gestão da produção em um cenário de Cadeia de Suprimentos. 2002. 125f. Dissertação (Mestrado em Engenharia de Produção) - Escola de Engenharia de São Carlos da Universidade de São Paulo, São Carlos, 2002.

BROWERSOX, D.J; CLOSS, D. J. Logística Empresarial. São Paulo: Atlas, 2001.

CALLADO, A.A.C; CALLADO, A.L.C. Mensuração de desempenho e agronegócio. In: Callado, A,A.C. (Org.). Agronegócio. 3ed. São Paulo: Atlas, 2010. p. 136-149.

CHEN, I.J.; PAULRAJ, A. Towards a theory of supply chain management: the constructs and measurement. Journal of Operational Management, Cambridge, v. 22, n. 2, p. 119-150, February, 2004.

CONCEIÇÃO, S. V.; QUINTÃO, R. T. Avaliação do desempenho logístico da cadeia brasileira de suprimentos de refrigerantes. Gestão e Produção, São Carlos, v. 11, n. 3, p. 441-453, September-December, 2004.

COOPER, M.C; ELLRAM, L. M. Characteristics of supply chain management and the implications for purchasing and logistics strategy. The International Journal of Logistics Management, Cambridge, v. 4, n. 2, p. 13-24, Jully-December, 1993.

COOPER, M.C; LAMBERT, D.M.; PAGH, J.D. Supply chain management: more than a new name for logistics. The international journal of logistics management, Cambridge, v. 18, n. 1, p. 67-89, January-April, 1997.

ELROD,C.; MURRAY, C.; BANDE, S. A review of performance metrics for supply chain management. Engineering Management Journal, Huntsville, v. 25, n. 3, p. 39-50, September-December, 2013.

GIL, A.C. Como elaborar projetos de pesquisa. 4ed. São Paulo: Atlas, 2008.

GOLDENBERG, M. A arte de pesquisar: como fazer pesquisa qualitativa em ciências sociais. São Paulo: Editora Record, 2002. 
GUNASEKARAN, A; PATEL C; MCGAUCHEY, R.E. A framework for supply chain performance measurement. International Journal of Operations \& Production Management, Cambrigde, v. 87, n. 3, p. 333347, February, 2004.

HOFMAN, D. The hierarchy of supply chain metrics. Supply Chain Management Review, Evanston, v. 8, n. 6, p. 28-37, September, 2004.

LEVIN, J. Estatística aplicada a ciências humanas. 2. ed. São Paulo: Harbra, 1987.

LOVEJOY, W.S; TSAY, A.A. Quantity Flexibility Contracts and Supply Chain Performance. Manufacturing \& Service Operations Management, Catonsville, v. 1, n. 2, p. 89-111, February, 1999.

LUCHT, RR. Escala de mensuração do desempenho de cadeias de suprimentos: a proposição de um modelo para a indústria de autopeças brasileira. In: SIMPÓSIO DE ADMINISTRAÇÃO DA PRODUÇÃO, LOGÍSTICA E OPERAÇÕES INTERNACIONAIS, 8., 2005, São Paulo. Anais... São Paulo: Fundação Getúlio Vargas, 2005. p 1-15. CD-ROM.

MENA, C.; HUMPHRIES, A,; CHOI, T.Y. Toward a theory of multi-tier supply chain management. Journal of Supply Chain Management, Chichester,, v. 49, n. 2, p. 58-77, April, 2013.

MORAIS, M.A.C. Proposta de um modelo para análise de cadeias de suprimentos de usinas siderúrgica: estudo de caso na Arcelormettal Tubarão Aços Planos. 2008. 206 f. Dissertação. (Mestrado em Engenharia de Produção) - Universidade Federal da Paraíba, João Pessoa, 2008.

NAJMI, M.; RIGAS, J.; FAN, I. A framework to review performance measurement systems. Business Process Management Journal, Cambridge, v. 11, n. 2, p. 109-122, March, 2005.

NEELY, A.; GREGORY, M.J.; PLATTS, K.W. Performance measurement system design. International Journal of Operations \& Production Management. Cambridge, v. 15, n. 4, p. 80-116, April, 1995. 
PIGATTO, G.; ALCÂNTARA, R. L.C. Relacionamento colaborativo nos canais de distribuição. In: QUEIROZ, Timóteo Ramos; ZUIN, Luís Fernando Soares (org.). Agronegócios:Gestão e Inovação. Rio de Janeiro, 2006. p. 129-166.

PIRES, S.R.I. Gestão da Cadeia de Suprimentos. São Paulo: Atlas, 2004.

RAFELE, C. Logistic service measurement: a reference framework. Journal of Manufacturing Technology Management, Cambridge, v. 15, n. 3, p. 280-290, April, 2004.

SHIN, H.; COLLIER, D.A.; WILSON, D. D. Supply chain orientation and supplier/buyer performance. Journal of Operations Management, Cambridge,v. 18, n. 3, p. 317-333, April, 2000.

Artigo recebido em: 06/05/2013

Aprovado em: 09/04/2014 